IOS Press

\title{
Editorial
}

\section{Web reasoning and rule systems}

\author{
Domenico Lembo ${ }^{\mathrm{a}}$ and Wolfgang Faber ${ }^{\mathrm{b}}$ \\ ${ }^{a}$ Department of Computer, Control, and Management Engineering, Sapienza University of Rome, Via Ariosto 25, \\ I-00185, Roma, Italy \\ E-mail:lembo@dis.uniromal.it \\ ${ }^{\mathrm{b}}$ School of Computing and Engineering, University of Huddersfield, Queensgate, Huddersfield HD1 3DH, \\ United Kingdom \\ E-mail:w.faber@hud.ac.uk
}

This special issue of the Semantic Web journal is a collection of contributions on the major topics discussed at the Seventh International Conference on Web Reasoning and Rule Systems (RR 2013). These topics include original research from all areas of Web Reasoning, with an emphasis on combinations with Rule Systems.

Combining Web Reasoning and Rule Systems embodies the main challenges to be faced by large-scale information systems that deal with heterogeneous, distributed, uncertain, changing, incomplete, and possibly contradictory information. The formalisms and systems need to provide expressiveness, yet be scalable; they need to be aware of context, yet be general; they need to cope with noise, yet provide crisp conclusions. These are just a few examples of the tensions that have to be resolved, and the International Conference on Web Reasoning and Rule Systems (RR) provides a major annual forum for discussions in these areas.

The seventh RR conference was held in Mannheim, Germany, during July 26-28, 2013. The conference program included presentations of 23 (full and short) papers that were selected out of 34 submissions. Each submission received at least 3 reviews.

The present special issue has been targeted to authors of papers presented at RR 2013 for preparing revised and extended versions of their conference contributions to be considered for publication in the Semantic Web Journal, but it was open to all submissions of papers addressing the conference topics.

We received seven submissions, two of which were extended versions of RR 2013 papers. Apart from one submission, which was rejected in pre-screening, each submission underwent a rigorous review process, involving at least three reviews per paper and two review rounds. Four submissions were eventually selected for this special issue, whereas one paper was rejected and another one was withdrawn.

- In Forest Logging: A Trace-Based Analysis of Large Rule-Based Computations, Terrance Swift presents a new way of profiling tabled computations involving rule bases interpreted using the well-founded semantics, which form the underpinnings of a number of rule-based Web Reasoning systems. The results obtained in this paper will allow for better performance analyses of Web Reasoning applications.

- In Sound, Complete and Minimal UCQ-Rewriting for Existential Rules, Mélanie König, Michel Leclère, Marie-Laure Mugnier, and Michaël Thomazo study query rewriting in the setting of existential rules, providing also a promising implementation that allows for more efficient ontology-based data access.

- In Probabilistic Description Logics under the Distribution Semantics, Fabrizio Riguzzi, Elena Bellodi, Evelina Lamma, and Riccardo Zese present a new way of integrating probabilities in ontologies. They also present a promising algorithmic approach for computing probabilities of queries on this kind of ontologies. This paper is an extended version of an RR 2013 contribution that was awarded the best paper prize. 
- In Coupling conceptual modeling and rules for the annotation of dramatic media, Vincenzo Lombardo, Cristina Battaglino, Antonio Pizzo, Rossana Damiano, and Antonio Lieto use ontologies and rules to model drama and characters therein, and they successfully use it for annotation purposes by means of reasoning.

We would like to thank all the authors for their contributions to this special issue and all reviewers (Leo- poldo Bertossi, François Bry, Maurice Bruynooghe, Claudia d'Amato, Pablo Gervás, Stijn Heymans, Erwan Jahier, Michael Kifer, Raghava Mutharaju, Alessandro Oltramari, Giorgio Orsi, Frantisek Simancík, Giorgos Stoilos, Umberto Straccia, and five others who opted to stay anonymous) for their valuable and careful review work that made the publication of this special issue a success. 